\title{
Closed-form solutions and free energy of hard-spin mean-field theory of a fully frustrated system
}

\author{
Alkan Kabakçıōlu, ${ }^{1,2}$ A. Nihat Berker, ${ }^{1,2}$ and M. Cemal Yalabık ${ }^{1}$ \\ ${ }^{1}$ Department of Physics, Bilkent University, Bilkent, Ankara 06533, Turkey \\ ${ }^{2}$ Department of Physics, Massachusetts Institute of Technology, Cambridge, Massachusetts 02139
}

(Received 10 December 1993)

\begin{abstract}
Closed-form solutions of the hard-spin mean-field theory equations for the antiferromagnetic Ising model on a triangular lattice, with or without an external field $H$, are obtained, showing the lack of order for $H=0$ and very good agreement with Monte Carlo data for the onset of order for nonzero $H$. A free energy calculation is developed, within the context of hard-spin mean-field theory, distinguishing between metastable solutions and true thermodynamic equilibrium.
\end{abstract}

PACS number(s): 05.70.Fh, 75.10.Nr, 64.60.Cn, 64.60.My

The recently introduced [1,2] "hard-spin mean-field theory" appears to be a rather promising new method of statistical physics [3]. Designed to conserve frustration, it has been quantitatively successful in yielding the orderings and phase boundaries of the fully frustrated antiferromagnetic triangular Ising model [1] (including the lack of finite-temperature phase transition at zero external field) and of the partially frustrated, ferromagnetically [1] or antiferromagnetically [2] stacked three-dimensional version of the model. Thus, unlike usual mean-field theory and other previous self-consistent theories, hardspin mean-field theory is sensitive to qualitative differences in ordering behavior between different spatial dimensions [1,2], in fact giving exact results [4] in $d=1$. Immediate further applications of the method to partially and fully frustrated square and cubic lattices has yielded phase diagrams that discerned up to 24 coexisting phases and 16 magnetization sublattices, and the novel phenomena of inclusive and exclusive coexistence lines [5]. Results have also been obtained on the competition between frustration and high-spin kinematics [6]. The method is also formulated for arbitrary types of local degrees of freedom [2].

Nevertheless, important questions on hard-spin meanfield theory have remained current. In the theory, the self-consistent equations for the thermodynamic densities are written directly from microscopic considerations. Thus, the question remains as to whether a variational principle exists that yields the equations from an optimization. In any case, a free energy calculation is needed to enable a choice when multiple solutions are found in the closed-form solution $[2,4,7]$ of the theory. Such a free energy calculation is presented in this article. This leads to the question of whether the closed-form solution and Monte Carlo implementation of the theory are equivalent. Interestingly, it is found in the work presented here, which is a detailed closed-form solution of hardspin mean-field theory, that the answer to the latter question is no: Monte Carlo hard-spin mean-field theory calculates a distribution of local magnetizations and yields [1], for example, correctly for the three-state Potts model, the second-order phase transition in two dimensions and the first-order phase transition in three dimensions.
Therefore, the self-consistent functional equation for the distributions of magnetizations is needed and given at the end of this study. Application of this functional selfconsistency should lead, in closed-form, in the direction of Monte Carlo hard-spin mean-field theory.

Consider the antiferromagnetic Ising model on the triangular lattice, with Hamiltonian

$$
-\beta \mathcal{H}=-J \sum_{\langle i j\rangle} s_{i} s_{j}+H \sum_{i} s_{i}
$$

where $\langle i j\rangle$ denotes the summation over all nearestneighbor pairs of sites, a spin $s_{i}= \pm 1$ is located at each lattice site $i$, and $J \geq 0$. Thus, the interactions of the system [the first term in Eq. (1)] are fully frustrated. The hard-spin mean-field-theory self-consistent equation for the magnetizations is $[2,7]$

$m_{i}=\sum_{\left\{s_{j}= \pm 1\right\}}\left[\prod_{j} p\left(m_{j} ; s_{j}\right)\right] \tanh \left(-J \sum_{j} s_{j}+H\right]$,

where the product and sum over $j$ runs over all sites neighboring site $i$, and the single-site probability distribution $p\left(m_{j} ; s_{j}\right)$ is $\left(1+m_{j} s_{j}\right) / 2$. Thus, the spin at each site is affected by the anti-aligning field due to the full (i.e., hard) spin each of its neighbors. The above is a set of coupled equations for all the local magnetizations. A Monte Carlo treatment [1] of the hard-spin mean-fieldtheory equations involves (1) the choice of a site $i$, (2) the fixing of each neighboring $s_{j}$ as \pm 1 for $r \lessgtr m_{j}$, where $r$ is a random number in the interval $[-1,1]$, and (3) the updating of $m_{i}$ as $\tanh \left(-J \sum_{j} s_{j}+H\right)$. Then, the process is repeated, starting with step (1). Excellent results are obtained with a quasinegligible computational effort [1].

The hard-spin mean-field-theory equations (2) can also be solved in closed-form, numerically. We have obtained such a solution by fixing the local magnetizations $\left\{m_{i}\right\}$ to values for three sublattices $\left\{m_{1}, m_{2}, m_{3}\right\}$. A solution using 81 sublattices $\left\{m_{1}, \ldots, m_{81}\right\}$ reduces to the threesublattice solution. The stable solutions (thick curves in Figs. 1 and 2) are obtained by iterating repeatedly Eqs. (2) successively applied to each sublattice magnetization. The unstable solutions (thin curves in Figs. 1 and 2) are 
obtained by iterating repeatedly a Newton-Raphson procedure on Eqs. (2). Among the stable solutions, it is found that a uniform solution $\left(m_{1}=m_{2}=m_{3}\right)$ is supplemented at low temperatures by a threefold symmetrybroken solution ( $m_{1} \neq m_{2}=m_{3}$ and permutations).
A higher level of approximation is

$$
m_{1}=\sum_{\left\{s_{j}= \pm 1\right\}}\left[\prod_{j} p\left(m_{j} ; s_{j}\right)\right] u_{1}\left(\left\{s_{j}\right\}\right)
$$

with

$$
\begin{aligned}
u_{1}\left(\left\{s_{j}\right\}\right)= & \sum_{\left\{s_{i}\right\}} s_{1} \exp \left[-\beta \mathcal{H}\left(\left\{s_{i}, s_{j}\right\}\right)\right] / \sum_{\left\{s_{i}\right\}} \exp \left[-\beta \mathcal{H}\left(\left\{s_{i}, s_{j}\right\}\right)\right], \\
-\beta \mathcal{H}\left(\left\{s_{i}, s_{j}\right\}\right)= & -J\left(s_{1} s_{2}+s_{2} s_{3}+s_{3} s_{1}\right)+H\left(s_{1}+s_{2}+s_{3}\right) \\
& \quad-J s_{1}\left(s_{4}+s_{5}+s_{6}+s_{7}\right)-J s_{2}\left(s_{7}+s_{8}+s_{9}+s_{10}\right)-J s_{3}\left(s_{10}+s_{11}+s_{12}+s_{4}\right),
\end{aligned}
$$

where the sites $i=1,2,3$ form an elementary triangle of the lattice, and $j=4,5, \ldots, 12$ runs over the nine sites neighboring this elementary triangle. An analogous equation applies for $m_{2}$ or $m_{3}$, obtained by replacing the subscripts 1 by 2 or 3 in the first two lines of Eq. (3). Thus, the statistical mechanics of a triplet of sites [as opposed to a single site, Eq. (2)] is done in the anti-aligning hard-spin fields of nine neighbors. These closed-form results are also shown in Figs. 1 and 2, and it is seen that the approximations are robust.

The occurrence of the symmetry-broken solutions, in the space of temperature $(1 / J)$ and relative field strength $(H / J)$, is shown in Fig. 3 for both levels of approximation. It is seen that no symmetry-broken solution occurs in the absence of external field ( $H=0)$, in agreement with Wannier's exact result [8] and in contrast to conventional mean-field theory. Also shown in Fig. 3 are data for the onset of order from an extensive Monte Carlo simulation study [9]. It is seen that these data points are remarkably close to the onset of the ordered solution here. Also shown in Fig. 3 is a lower temperature curve where the uniform solution crosses the unstable symmetry-broken

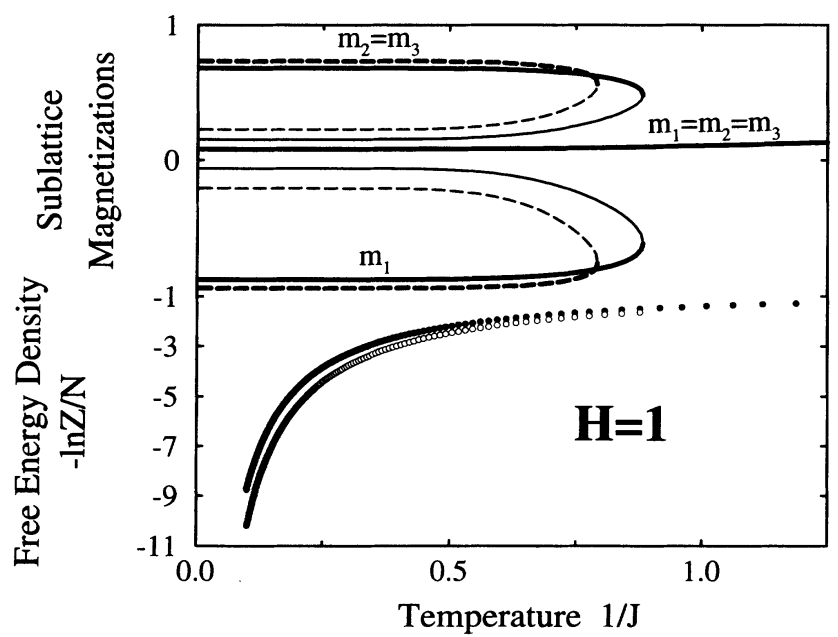

FIG. 1. Solutions from Eq. (3) of hard-spin mean-field theory, for $H=1$ (full curves). The stable and unstable solutions are, respectively, shown with thick and thin curves. The dashed curves are the solutions from the lower level of approximation of Eq. (2). Also shown are the calculated free energies per site, with dark circles (uniform solution) and open circles (symmetry-broken solution). solution and exchanges stability with it, as illustrated in Fig. 2.

In order to choose between the distinct solutions of the hard-spin mean-field-theory equations, a knowledge of the free energy of each solution is necessary. Accordingly, we consider the dimensionless free energy per site

$$
f(J, H)=-(1 / N) \ln \sum_{\{s\}} e^{-\beta H} .
$$

Its partial derivative with respect to inverse temperature is

$$
\partial f / \partial J=(1 / 2 N) \sum_{\langle i j k\rangle}\left(\left\langle s_{i} s_{j}\right\rangle+\left\langle s_{j} s_{k}\right\rangle+\left\langle s_{k} s_{i}\right\rangle\right) \text {. }
$$

The sum is over all nearest-neighbor triplets. The averages on the right-hand side are determined for each solution, by replacing $s_{1}$ with $s_{i} s_{j}+s_{j} s_{k}+s_{k} s_{i}$ on the righthand side of Eq. (3), once the sublattice magnetizations, and thereby the probability distributions $p\left(m_{j} ; s_{j}\right)$, have been determined self-consistently from Eq. (3). At high temperatures, $J \rightarrow 0$, the free energy of the uniform solution reduces to

$$
\begin{aligned}
f= & -\ln \left(e^{H}+e^{-H}\right) \\
& +(J / 2 N) \sum_{\langle i j k\rangle}\left(\left\langle s_{i} s_{j}\right\rangle+\left\langle s_{j} s_{k}\right\rangle+\left\langle s_{k} s_{i}\right\rangle\right) .
\end{aligned}
$$

At low temperatures, $J \rightarrow \infty$, the free energy of the symmetry-broken solution reduces to

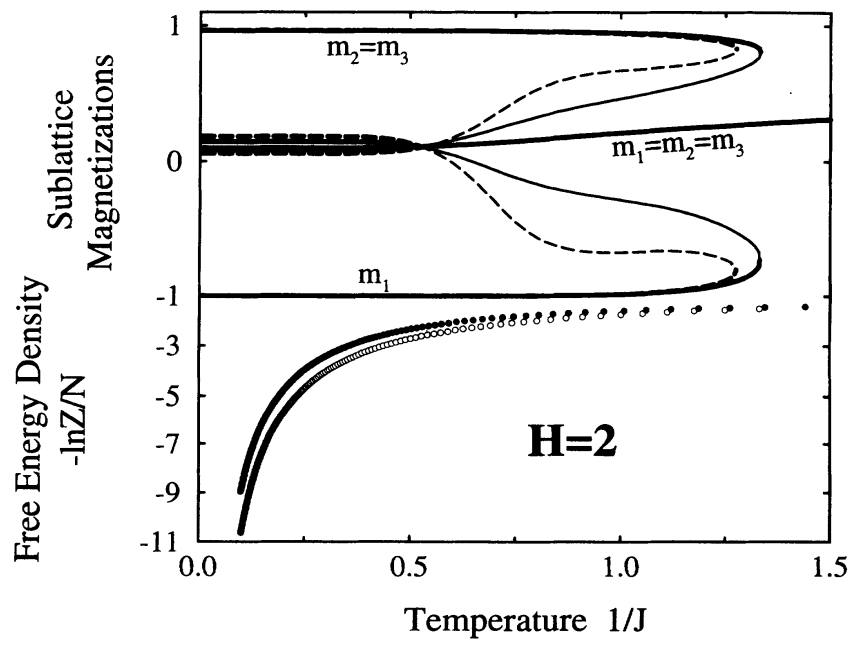

FIG. 2. Same as in Fig. 1, but for $H=2$. 


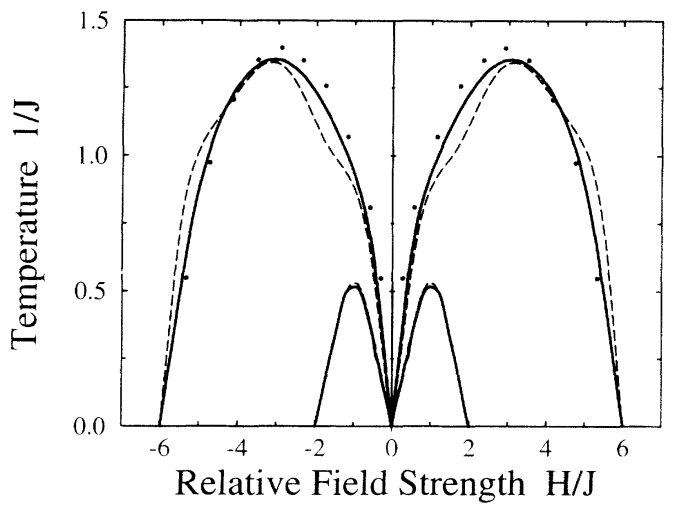

FIG. 3. The upper curve bounds the regions of temperature $(1 / J)$ and relative field strength $(H / J)$ where a symmetrybroken solution occurs. The dark circles are the Monte Carlo simulation data for the onset of symmetry breaking, from Ref. [9]. The lower curve shows where the uniform solution and the unstable symmetry-broken solution cross and exchange stability, as illustrated for $H=2$ in Fig. 2. The full and dashed curves are obtained from the two levels of approximations of Eqs. (3) and (2), respectively.

$$
\begin{aligned}
f= & (J / 2 N) \sum_{\langle i j k\rangle}\left(\left\langle s_{i} s_{j}\right\rangle+\left\langle s_{j} s_{k}\right\rangle+\left\langle s_{k} s_{i}\right\rangle\right) \\
& -(H / 6 N) \sum_{\langle i j k\rangle}\left(\left\langle s_{i}\right\rangle+\left\langle s_{j}\right\rangle+\left\langle s_{k}\right\rangle\right)-S_{0}(H),
\end{aligned}
$$

where $S_{0}(H)$ is the ground-state entropy per site under uniform field $H$. For large $|H|$, the sublattice magnetizations of the symmetry-broken phase fully saturate at $|\boldsymbol{H}| \ll J \rightarrow \infty$ to $\pm(1,1,-1)$ and permutations, so that the ground-state entropy $S_{0}(H)$ is zero. However, for low $|\boldsymbol{H}|$, these sublattice magnetizations do not fully saturate, and the system has a finite ground-state entropy $S_{0}(H)$. As a trial, we use the entropy of free spins under fields $\left(H_{1}, H_{2}, H_{3}\right)$ causing magnetizations $\left(m_{1}, m_{2}, m_{3}\right)$, namely,

$$
\begin{aligned}
S_{0}(H)=\ln 2-\left(\frac{1}{6}\right) \sum_{i=1,2,3} & {\left[\left(1+m_{i}\right) \ln \left(1+m_{i}\right)\right.} \\
& \left.+\left(1-m_{i}\right) \ln \left(1-m_{i}\right)\right] .
\end{aligned}
$$

The thermodynamic densities in Eq. (6) are, of course, calculated at the uniform solutions of Eq. (3), and the thermodynamic densities in Eqs. (7) and (8) are calculated at the symmetry-broken solutions of Eq. (3). Thus, the free energies of the uniform and symmetry-broken solutions are obtained by integrating Eq. (5) at constant $H$ from high and low temperatures, respectively, as shown in Fig. 4, and adding the limiting free energies of Eqs. (6) or (7), respectively.

The calculated free energies for $H=2$ are shown in Fig. 2. The symmetry-broken solution has the lower free energy at low temperatures, in its entire range of existence. The two free energies, calculated from opposite temperature extremes, meet at the point of appearance of the symmetry-broken solution. Note that there is no built-in requirement for this occurrence, as will be seen below. The symmetry-broken magnetizations in Fig. 2

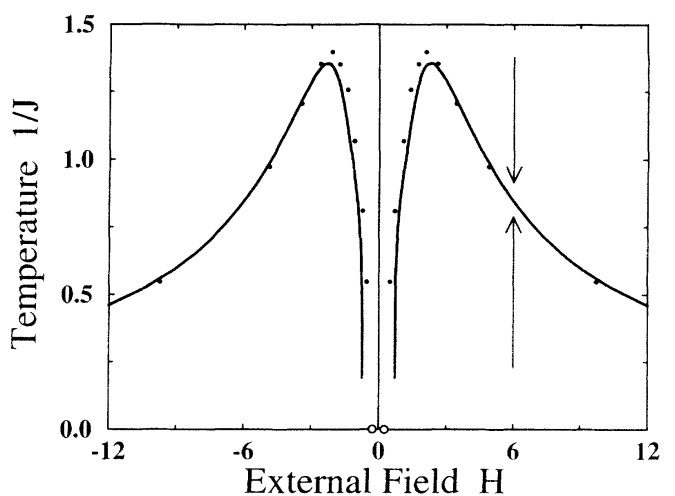

FIG. 4. The curves bound the region in temperature $(1 / J)$ and field strength $(H)$ where a symmetry-broken solution occurs, as obtained from Eq. (3). The data points are from Monte Carlo simulation (dark circles, Ref. [9]) and finite-size scaling (open circles, Ref. [10]). The arrows show the paths of integration of the uniform (upper arrow) and symmetry-broken (lower arrow) solutions.

essentially saturate at low temperature, so that the zerotemperature entropy term discussed above is negligible for this case.

The free energy results shown in Fig. 2 are qualitatively reproduced for other values of $H$, except when the low-temperature symmetry-broken magnetizations do not fully saturate, which occurs for low values of $|H|$. This situation is illustrated for $H=1$ in Fig. 1. In this case, the free energy of the symmetry-broken solution is again lower in its entire range of existence, but, as calculated with $S_{0}(H)$ from Eq. (8), it does not meet the free energy of the uniform solution at the point of appearance. In fact, the entropy $S_{0}(H)$, which is the logarithm of the number of microscopic states consistent with $\left(m_{1}, m_{2}, m_{3}\right)$ divided by the number of sites, is overestimated by Eq. (8), since the constraints imposed by $J \rightarrow \infty$ are ignored. Accordingly, $S_{0}(H)$ from Eq. (8) reduces for $H=0$ to the free-spin value of $\ln 2$, whereas Wannier's exact result [8] gives 0.323 . When the expression of Eq. (8) is scaled to match 0.323 at $H=0$, the free energies cross somewhat below the point of appearance.

The above implies a first-order phase transition with critical correlations in one of the coexisting phases, namely, in the symmetry-broken phase. Now we note that when the hard-spin mean-field theory Eqs. (2) or (3) for the local magnetizations $\left\{m_{i}\right\}$ are solved in terms of sublattice-wise uniform magnetizations, an orderparameter jump will always be obtained at the phase transition of the threefold permutation-symmetric (three-state Potts) ordering, because of the third-order term in the small order-parameter expansion of the equation. What is remarkable here is that the equations come as close to a second-order phase transition as they possibly can, by putting the appearance of the symmetrybroken solution at the order-parameter jump, while also giving the position of the transition at its correct value, as compared with Monte Carlo simulation data [9] (Figs. 3 and 4).

Monte Carlo hard-spin mean-field theory with Eq. (1) 
treats the local magnetization $\left\{m_{i}\right\}$ independently and yields [1] the expected second-order phase transition of this ordering, which is in the universality class of the two-dimensional three-state Potts model. Moreover, Monte Carlo hard-spin mean-field theory also yields [1] the expected first-order phase transition of this ordering in the stacked version of this system, which is in the universality class of the three-dimensional three-state Potts model. The success of Monte Carlo hard-spin mean-field theory must be due to the fact that, in treating local magnetizations, the theory incorporates correlations between different sites. Accordingly, to include this effect in a closed-form solution, the hard-spin mean-field theory for the distribution $D_{i}\left(m_{i}\right)$ of local magnetizations $m_{i}$ at site $i$ must be considered. This equation is

$D_{i}\left(m_{i}\right)=\int\left[\prod_{j} d m_{j} D_{j}\left(m_{j}\right)\right] \delta\left(m_{i}-M_{i}\left(\left\{m_{j}\right\}\right)\right)$,

where $M_{i}\left(\left\{m_{j}\right\}\right)$ is the right-hand side of Eq. (2) or Eq.
(3), depending on the chosen level of approximation. This distribution hard-spin mean-field theory [Eq. (9)] and Monte Carlo hard-spin mean-field theory [1] also open the door to the possibility of non-mean-field critical exponents, since one is in effect doing Landau theory with infinitely many order parameters. This possibility should be further studied. The imposition of uniformity, on the other hand, dictates standard mean-field exponents, since the small order-parameter analysis is then standard Landau theory.

We are thankful to A. Naqvi and R. R. Netz for useful discussions. A.N.B. thanks the Scientific and Technical Research Council of Turkey (TÜBITAK) for a travel grant and the members of the Physics Department of Bilkent University for their hospitality. This research was supported by the U.S. Department of Energy under Grant No. DE-FG02-92ER45473.
[1] R. R. Netz and A. N. Berker, Phys. Rev. Lett. 66, 377 (1991).

[2] R. R. Netz and A. N. Berker, J. Appl. Phys. 70, 6074 (1991).

[3] A. N. Berker, A. Kabakçıōlu, R. R. Netz, and M. C. Yalabık, Doḡa Tr. J. Phys. 18, 354 (1994).

[4] J. Banavar, M. Cieplak, and A. Maritan, Phys. Rev. Lett. 67, 1807 (1991).
[5] R. R. Netz, Phys. Rev. B 46, 1209 (1992).

[6] R. R. Netz, Phys. Rev. B 48, 16113 (1993).

[7] R. R. Netz and A. N. Berker, Phys. Rev. Lett. 67, 1808 (1991).

[8] G. H. Wannier, Phys. Rev. 79, 357 (1950).

[9] B. D. Metcalf, Phys. Lett. 45A, 1 (1973).

[10] H. W. J. Blöte and M. P. Nightingale, Phys. Rev. B 47, 15046 (1993). 